\title{
El kumi-uchi desde la perspectiva de la Kaze no Ryu
}

Luis Nogueira Serrano

European Bugei Society

Recibido 22/07/2010 - Aceptado 30/09/2010

\section{Resumen}

El kumi-uchi, literalmente "agarrar y golpear", es un arte de combate tradicional japonés basado en técnicas que se realizan fundamentalmente sin armas. Como tal, se considera que surgió como complemento a las artes marciales principales del samurái, para hacer frente a situaciones difíciles en las que este perdía sus armas más poderosas. El presente trabajo estudia el arte del kumi-uchi desde la perspectiva de la escuela Kaze no Ryu. Se analiza el significado y extensión del término, sus aspectos fundamentales, tipos, dimensión y evolución. Del mismo modo, se ubica el kumi-uchi dentro del currículo marcial de la Kaze no Ryu y se reflexiona sobre su importancia dentro de la formación del practicante de artes marciales.

Palabras clave: Artes marciales, Japón, escuelas tradicionales, koryu, taijutsu.

\section{Kumi-uchi from the Kaze no Ryu perspective}

Abstract: Kumi-uchi, literally "grasp and strike", is a traditional Japanese fighting art mainly based on non-armed techniques. As such, kumi-uchi is consider to have emerged as a complement to the main samurai martial arts, so as to face those difficult situations in which the samurai lost his most powerful weapons. This paper studies the art of kumiuchi from the Kaze no Ryu school standpoint. The meaning and extension of kumi-uchi, its main aspects, types, dimension and evolution are analyzed. Similarly, we place kumiuchi into the Kaze no Ryu martial curriculum and reflect on its importance in martial artists' education.

Key words: Martial arts, Japan, traditional schools, koryu, taijutsu.

\section{O kumi-uchi na perspectiva de Kaze no Ryu}

Resumo: 0 kumi-uchi, literalmente "agarrar e golpear", é uma arte de combate tradicional japonesa baseada em técnicas que se realizam fundamentalmente sem armas. Como tal, se considera que surgiu como complemento das artes marciais principais do samurai, para fazer frente a situações difíceis quando que este perdia as suas armas mais poderosas. 0 presente trabalho estuda a arte do kumi-uchi na perspectiva da escola Kaze no Ryu. Analisa-se 0 significado e a extensão da terminologia, os seus aspectos fundamentais, os tipos, a dimensão e a evolução. Do mesmo modo, se junta o kumi-uchi dentro do currículo marcial da Kaze no Ryu e se reflecte sobre a sua importância dentro da formação do praticante de artes marciais.

Palavras-chave: Artes marciais, Japão, escolas tradicionais, koryu, taijutsu. 


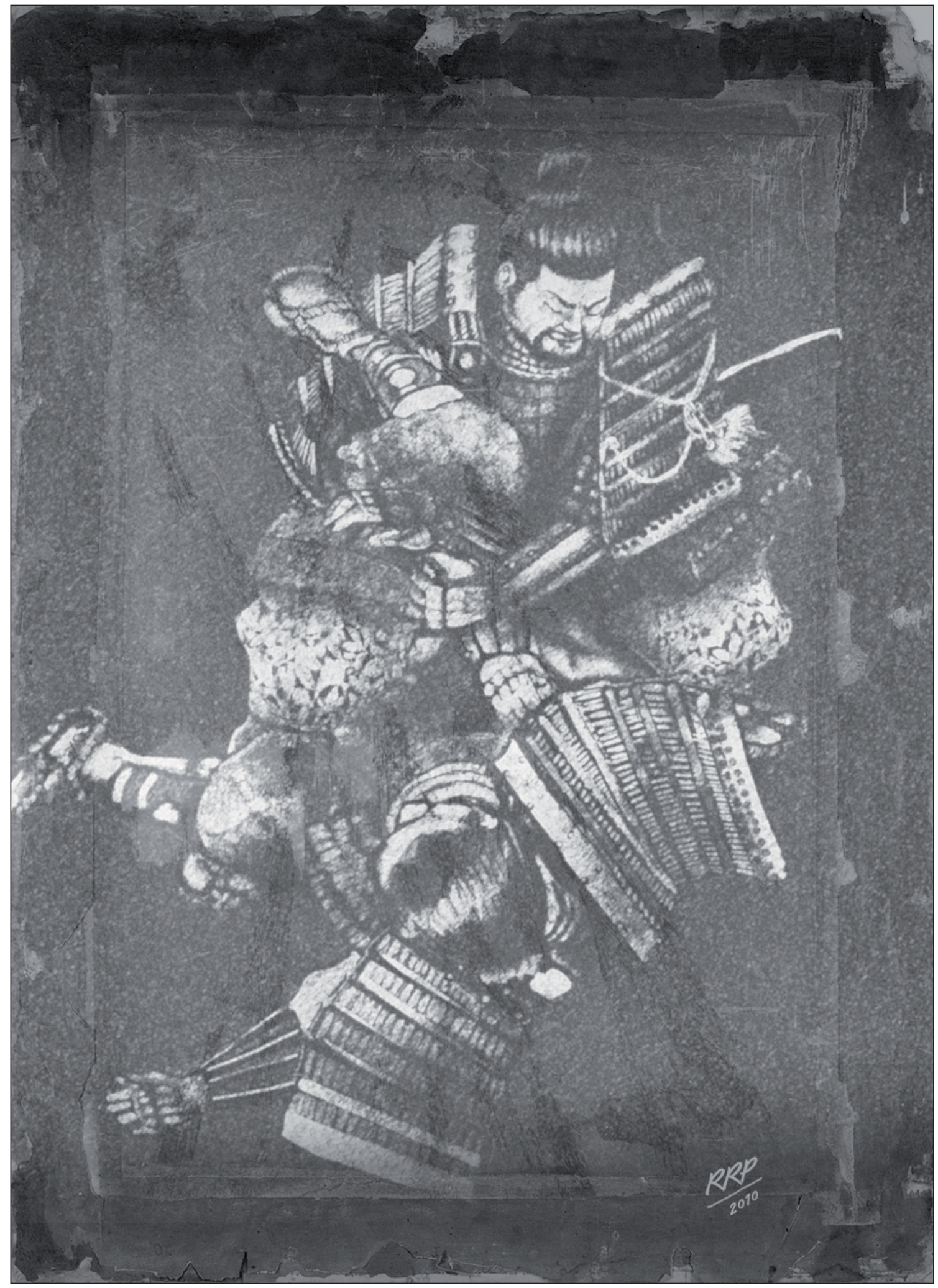

Kumi-uchi, por Rebeca Roca Pritchard (rebeca@bugel.eu).

Todas las ilustraciones son cortesía y propiedad de la European Bugei Society. 


\section{El kumi-uchi desde la perspectiva de la Kaze no Ryu}

Luis Nogueira Serrano

European Bugei Society

\section{Introducción}

El kumi-uchi es una de las artes tradicionales japonesas de combate más desconocidas que existen. Este desconocimiento se debe, en gran medida, a que el paso de los siglos ha ido relegando al olvido a esta antigua práctica, que se ha visto reemplazada -como muchas otras- por artes marciales más modernas. Actualmente, el kumi-uchi forma parte del currículo de diversas escuelas tradicionales de artes marciales japonesas (koryu) (Mol, 2001), como la Takenouchi Ryu, Yagyu Shingan Ryu o Sosuishi Ryu, por citar algunas de las más destacadas. En el currículo marcial de la Kaze no Ryu, el kumi-uchi es una de las materias incluidas dentro del taijutsu (literalmente, arte, técnica o habilidad del cuerpo ${ }^{1}$ ). A su vez, el taijutsu es una de las 18 materias estudiadas dentro del Bugei Juhappan.

El Bugei Juhappan supone uno de los primeros intentos de clasificación de las disciplinas militares del Koryu. Como bien señala Gatling (2010: 120), no existe una clasificación estandarizada de estas 18 materias, por lo que el listado puede variar de unas escuelas a otras. Se trata de una ordenación que no se basa en un solo criterio, sino en criterios dispares que surgieron a lo largo de la historia en función de las particulares visiones que tenían los propios clasificadores respecto a las artes marciales. Las diferencias entre estas clasificaciones, analizadas de forma genérica, no suponen más que simples apreciaciones particulares de cada autor, y lo central es valorar el intento de clasificar las artes con el objeto de sistematizar una comprensión general de todas las koryu.

Las primeras clasificaciones de las artes militares que influyeron en Japón llegaron de China y se remontan alrededor del 1900 a.C. durante los periodos de Ken-En-Tei. Sin embargo, estas clasificaciones y sus sucesivas, por mucho que supusieran una influencia posterior en las artes militares japonesas, no fueron más que posibles precursoras de dichas clasificaciones. No fue hasta el periodo Edo (1603-1868 d.C.), cuando Hirayama Gyozo, también inspirado en fuentes chinas, escribió la obra Bugei Juhappan Ryakosetsu, siendo este el verdadero precursor del término (Friday \& Humitake, 1997) que fue sucedido por múltiples clasifica-

1 También son comunes otras traducciones menos exactas como "artes sin armas", "combate cuerpo a cuerpo", etc. 
ciones, en particulares intentos de responder mejor a las muchas realidades en cada koryu.

Otro aspecto importante sobre el Bugei Juhappan es su número, el 18, que ya se contiene en su propia denominación (18 materias estudiadas en el bugei). Casualmente, en casi todas las versiones se hizo un esfuerzo muy especial por parte de los compiladores en clasificarlo en 18 materias. En algunos casos se clasificó en 36 , que es dos veces 18 . El número 18 , por sus singularidades matemáticas, es un número considerado mágico en diferentes culturas, aunque por el momento nos son desconocidas las razones concretas que llevaron al establecimiento y perpetuación de este número en las artes marciales clásicas japonesas.

Con todo, existen otras clasificaciones que han pasado por alto la característica del número 18 , tanto que cada escuela tiene finalmente su propio sistema de clasificación y son pocas las escuelas que aún mantienen la identidad del Bugei Juhappan en su más amplio sentido y generalmente por causas compilatorias.

En la Tabla 1 se muestra una organización sencilla de las materias más conocidas estudiadas en la escuela Kaze no Ryu Bugei.

\begin{tabular}{|c|c|}
\hline Grupo & Artes/Armas \\
\hline 1. Taijutsu & $\begin{array}{l}\text { Kumi-uchi } \\
\text { Jujutsu } \\
\text { Aikijujutsu } \\
\text { Koppojutsu } \\
\text { Kenpo }\end{array}$ \\
\hline 2. Bujutsu & $\begin{array}{l}\text { Kenjutsu (daisho katana y wakizashi) } \\
\text { laijutsu } \\
\text { Battojutsu } \\
\text { Tantojutsu } \\
\text { Yarijutsu (fuko y mago yari) } \\
\text { Naginata } \\
\text { Bojutsu } \\
\text { Jojutsu } \\
\text { Tanbojutsu } \\
\text { Shurikenjutsu } \\
\text { Kyujutsu } \\
\text { Juttejutsu } \\
\text { Kusari Jutsu } \\
\quad \text { Kusarigama } \\
\quad \text { Kusarifundo } \\
\quad \text { Manriki Kusari } \\
\text { Hojojutsu }\end{array}$ \\
\hline
\end{tabular}

No obstante, la escuela Kaze no Ryu posee una clasificación propia de 33 disciplinas físicas y 44 disciplinas mentales, lo que hace un total de 77 materias de estudio. La documentación de todas ellas es una labor ardua, pues muchas materias poseen subclasificaciones, y excede las posibilidades y pretensión del presente trabajo. Señalamos simplemente, a modo de ejemplo que el hojojutsu ("arte del método de las cuerdas", estudio de las formas clásicas de aprisionar a un oponente con cuerdas), dispone del estudio de torinawa, hayanawa, kage- 
nawa y gyakko². Además de esto existen otras disciplinas como el shibaru (que puede traducirse por "restringir" o "retener"), que visa el uso de las cuerdas pero en condiciones y objetivos diferentes. Igualmente, dentro de la escuela existen materias de conocimiento transversal, como podría ser el atemi no gikko (disciplina orientada al estudio de las formas de impacto), que más allá de ser una materia independiente posee aplicación en todas las materias del taijutsu.

Como se ha señalado, el kumi-uchi forma parte del taijitsu. Etimológicamente proviene de la junción de los siguientes ideogramas (kanji) japoneses:

\section{組 Kumi, que se traduce por las ideas de agarrar, apresar, asegurar. 打 Uchi, que se entiende como golpear, batir.}

EL KUMIUCHI ESTÁ TOTALMENTE VINCULADO AL AGARRE. LA MECÁNICA DE LOS AGARRES PERMITE ADOPTAR ESTRATEGIAS QUE DIFICULTAN EL MOVIMIENTO DEL

ADVERSARIO E IMPOSIBILITAN

QUE DESARROLLE UNA

REACCIÓN EFICAZ.

Fotografías de estudio por Javier Pastor Gimeno (www.valenciafotografos.com).

Por tanto, y en su literalidad, el origen etimológico de esta combinación alude a la idea de "agarrar y golpear". No obstante, la cuestión etimológica no ha de conducir al reduccionismo de pensar en el kumi-uchi como un arte basado únicamente en agarres y golpeos, pues, aunque primitiva, su riqueza en cuanto a otras técnicas de lucha es más que palpable. Como desarrollaremos más adelante, el kumi-uchi se compone también de técnicas de proyección, de control, etc. Igualmente, comprende el estudio de técnicas con armas apropiadas para el enfrentamiento a muy corta distancia, tales como el jutte, el tanbo, el cuchillo (tanto 0 aikuchi), y otras menos frecuentes como la kozuka, el hishigi, etc. ${ }^{3}$ En este sentido,

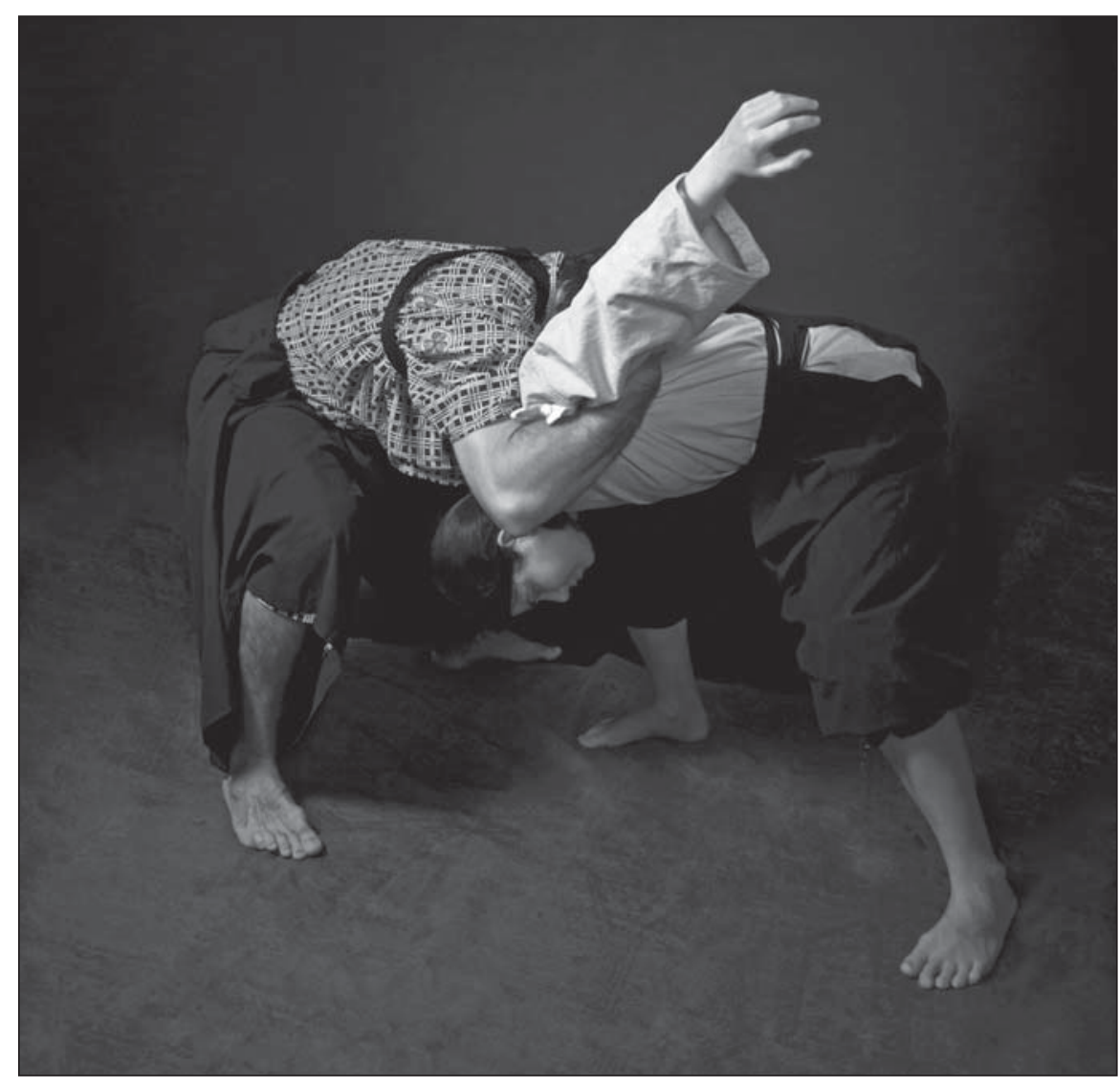

\footnotetext{
2 Tori proviene de toru, que significa "coger", "sujetar" y nawa significa "cuerda" (mismo kanji que el anterior jo de hojojutsu). Por tanto, puede ser traducido como "cuerda de sujeción o de presa". Haya significa "rápido", y por tanto hayanawa significa "cuerda rápida". Kage significa gancho, siendo por kagenawa una "cuerda con ganchos". Por último, gyakko son las formas clásicas estudiadas para apresar y que no poseen preparación de nudos en la cuerda como sucede en las disciplinas anteriores.

3 El jutte 0 jitte es un arma que utilizaba el samurái para apresar al oponente. Consistía en una empuñadura y prolongación metálicas con un gancho próximo a la empuñadura que permitía el desarme y atrapar los miembros del oponente. Por su parte, tanto el tanbo como el hishigi eran armas de madera de pequeño tamaño. El tanbo (o tenkai, como se denomina en la Kaze no Ryu) es una porra de entre 30 y $50 \mathrm{~cm}$. El hishigi era de un tamaño menor aún. Los kozuka
} 
comparte las mismas características que el conjunto de artes del taijitsu. Por último, el campo de aplicación del kumi-uchi no se reduce a técnicas empleadas en el combate de pie, sino que también incluye un amplio abanico de técnicas de combate en el suelo.

\section{Cuestiones fundamentales}

El medio en y para el que se desarrolló el kumi-uchi fue el campo de bataIla. La necesidad de poseer una serie de habilidades para el combate a cuerpo a muy corta distancia surgió desde el primer momento en que los guerreros fueron conscientes de la posibilidad de perder sus armas principales (arco, lanza, espada, naginata, etc.) en el fragor del combate, y tener que seguir combatiendo de la mejor manera posible. En estas circunstancias, la mejor opción para el guerrero era reducir al máximo la distancia respecto al oponente, de modo que este no pudiese utilizar eficazmente las armas que posiblemente él sí portaba.

Por este motivo, las técnicas de kumi-uchi siempre comienzan con una embestida violenta. La necesidad de acortar distancias de forma rápida y sorpresiva se acomete con ferocidad, embistiendo enérgica y decididamente con el cuerpo para dañar 0 al menos desequilibrar al oponente. Las zonas corporales más características con las que impactar son la cabeza y los hombros, que actúan a modo de ariete accediendo a zonas menos protegidas del rival. Así, las técnicas de ataque, y particularmente de impacto del kumi-uchi difieren de las que se suelen encontrar en formas modernas de artes marciales, que buscan el impacto con otras partes corporales como la mano o el pie. Este tipo de impactos, obviamente, tienen sentido en un combate en el que los adversarios no llevan una sólida armadura, pero no en los combates con armadura propios de la época feudal japonesa.

Para analizar de forma pormenorizada el impacto que se produce con la embestida resulta pertinente conocer algunas características y partes de la armadura japonesa (yoroi). Entre los muchos elementos que la componen, a los fines del presente estudio resultan especialmente relevantes aquellos que protegen la zona alta del cuerpo, esto es, el casco (kabuto), la máscara (menpo) y las protecciones de hombros y brazos (sode).

El kabuto era uno de los elementos más peculiares y característicos de la armadura samurái. Dependiendo de las épocas y del rango del samurái portador del mismo, los estilos eran claramente diferentes, pudiendo observar una notable evolución a lo largo del tiempo (Augusto $\&$ Galende, s.f.). Estaba compuesto por varias piezas, siendo la principal el casco en sí (hachi), con forma de cúpula y que se encargaba de proteger el cráneo. Otras partes del casco eran la visera (maezashi), el detalle frontal o cresta del casco (maedate), el cubrenuca (shikoro) y las lengüetas laterales situadas entre la visera y el cubrenuca (fukigaeshi). El shikoro descendía desde el hachi hacia el cuello por los costados y la espalda, y habitualmente se componía de 3 a 7 filas de entre 100 y 138 placas metálicas que protegían el cuello de posibles ataques laterales y posteriores. En conjunto, el kabuto era una sólida estructura ideada para proteger al guerrero, pero que también ante la necesidad podía convertirse en una contundente arma de ataque.

son pequeños filos de metal que se solían ubicar en la saya (vaina) de la katana (para una mayor información, véase Mol, 2003). 
El menpo es la máscara o elemento de protección facial característico de la armadura japonesa, popularizado en tiempos recientes a nivel mundial por su aparición en la serie de películas de El Silencio de los Corderos. Lejos de anécdotas, y al margen de su función como elemento intimidatorio, el menpo tenía un fin fundamental que era salvaguardar el rostro ante los ataques del enemigo, y secundariamente en muchos movimientos de impacto como los que caracterizan al kumi-uchi.

Por último, se conoce como sode al elemento protector de hombros y brazos. Su construcción se realizaba tejiendo con cuero 0 seda pequeñas piezas que conformaban bandas rectangulares 0 cuadradas. Dependiendo de su tamaño recibía las denominaciones de 0-sode, chu-sode o ko-sode, siendo las 0-sode las más grandes y las ko-sode las más pequeñas. La parte superior de estas protecciones, una pieza metálica habitualmente adornada, se denominaba kamuri-ita. El sode, al igual que los elementos anteriores, servía de protección en los impactos, y secundariamente también podía servir de arma.

Gracias a estos elementos, la embestida lograba el doble objetivo de acortar la distancia y utilizar el cuerpo protegido para atacar al oponente. Como hemos señalado, se trata de un gesto rápido y violento, de gran potencia. Se realiza desde una posición flexionada y tensa, lista para el ataque. El centro de gravedad desciende gracias a una semiflexión de rodillas y a una mayor separación de los pies, promoviendo una base amplia y estable. Los pies también están semicontraídos, colocados ligeramente de modo transversal a la dirección del movimiento, al igual que las caderas, parte superior del tronco y parte inferior o hara ${ }^{4}$. El correcto asentamiento y alineación corporal genera una potencialidad óptima para desarrollar la máxima fuerza explosiva en el empuje.

Por tanto, la embestida pretende no sólo acortar la distancia sino hacer uso del cuerpo protegido por la armadura para destruir al oponente. Para ello requería de un acondicionamiento físico muy especial. Uno de los métodos de entrenamiento tradicionales consiste en el arrastre de pesados troncos, que se unían al cuerpo mediante cuerdas atadas a la altura de las caderas. Este entrenamiento con cargas máximas en régimen claramente anaeróbico proporciona un gran desarrollo de la coordinación muscular así como de la fuerza máxima. La Kaze no Ryu recopiló y estudió este y otros tipos de entrenamiento tradicionales, incluyéndolos como materia específica en el currículo de la escuela con el nombre de shinrin tais0 $0^{5}$. Así, el shinrin taiso busca el desarrollo de las capacidades físicas necesarias para la obtención de la eficiencia de movimientos.

Con todo, esta brutal embestida no era el fin del kumi-uchi. Su objetivo se centraba en la obtención de una ventaja clara en el combate para lograr la victoria.

\footnotetext{
4 El hara es un concepto fundamental dentro de la concepción japonesa del ser humano, y tiene una especial relevancia en el desarrollo de las artes marciales japonesas. La Kaze no Ryu incluye una materia especialmente orientada al desarrollo del hara, denominada haragei ("artes del vientre" o "artes de la zona abdominal"), de seis años de duración.

5 Shinrin taiso proviene de shinrin, que puede ser traducido por "naturaleza" y taiso, que puede ser entendido como "ejercicio" o "gimnasia". Aunque el sentido de "gimnasia natural" hace referencia a multitud de ejercicios oriundos propiamente de la escuela, el concepto es mucho más amplio, ya que abarca, entre otros, estudios de fisiología energética y salud.
} 


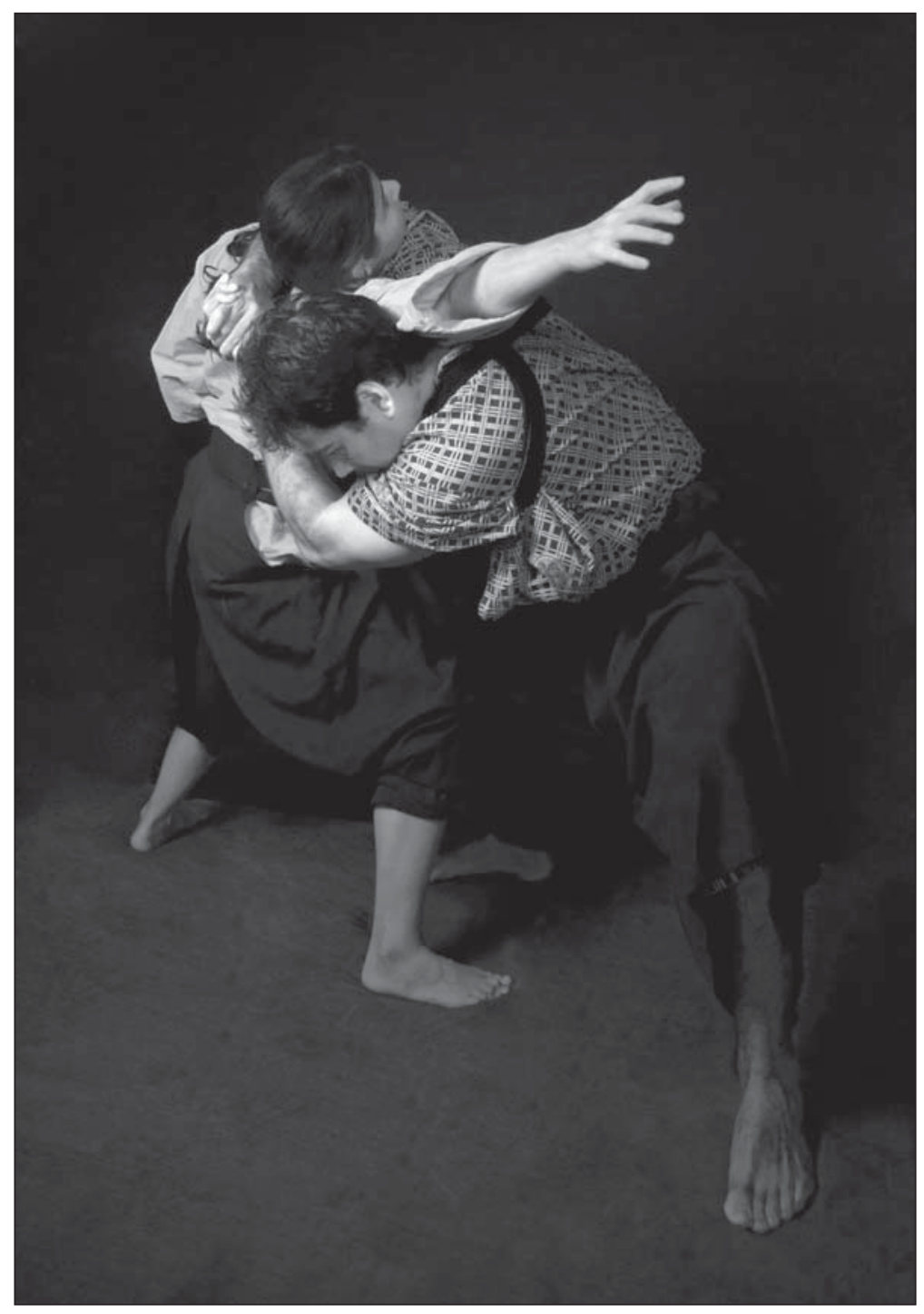

Estudiando las secuencias tradicionales de kumi-uchi, generalmente estas finalizaban con la sumisión del oponente y el uso del cuchillo (tanto 0 aikuchi) para rematarlo accediendo a las zonas vitales que la armadura dejaba desprotegidas.

La sumisión en el kumi-uchi se logra mediante poderosos agarres que inmovilizan y controlan al adversario, para continuar con una proyección, un control mediante luxación 0 una combinación de ambos procedimientos. Lógicamente, a pesar de su solidez el yoroi permitía un cierto grado de libertad de movimientos al guerrero para que este se desenvolviese en el combate, hecho que era aprovechado para aplicar luxaciones a articulaciones como el codo, hombro, muñeca, cuello, rodilla, etc., 0 estrangulaciones.

Un último aspecto a analizar dentro de los aspectos fundamentales del kumiuchi es el equilibrio. Como arte de combate, en el kumi-uchi se produce una resistencia y una lucha, una oposición de fuerzas que tratan de imponerse. Esta forma de combate es entendida como un triángulo en oposición que dibujan los cuerpos de ambos oponentes, aunque el concepto de "triangularización" es frecuente en los análisis de fuerzas del kumi-uchi. En este embate de

EN EL CONTROL QUE ILUSTRA LA FOTOGRAFÍA SE BUSCA NO SOLO LA ESTRANGULACIÓN

O LA LIMITACIÓN DEL MOVIMIENTO DEL BRAZO DEL ADVERSARIO, SINO TAMBIÉN IMPOSIBILITAR, A TRAVÉS DE

LA RELACIÓN DE ÁNGULOS CON EL HARA, LA REACCIÓN DEL OPONENTE. fuerzas, además de la posición (kamae), en el kumi-uchi cobra una especial importancia el kuzushi o desequilibrio. En las secuencias de kumi-uchi se evidencia una clara estrategia interna de combate que bascula entre el desequilibrio y el reposicionamiento favorable. El combate funciona como un laberinto que trata de atrapar al oponente en una senda sin salida que provoque su derrota. Es vital entender que en el kumi-uchi, al tratarse de un combate a muy corta distancia, el trabajo del kuzushi está orientado a "destruir" la estructura corporal del oponente, consiguiendo que este no pueda reaccionar de manera arbitraria.

En los procesos de equilibración, el aparato vestibular juega un papel fundamental. El aparato vestibular se encuentra en el oído interno, y está formado por diversas estructuras que transmiten señales nerviosas al sistema nervioso central relativas a aceleraciones, desaceleraciones e inclinaciones. Gracias a estas informaciones somos capaces de mantener el equilibrio de la postura y controlar nuestros movimientos. En el kumi-uchi muchos de los movimientos se orientan a confundir este sistema de equilibrio, a través de presas y movimientos que actúan sobre la cabeza del oponente, generando una dificultad añadida. 
En resumen, el kumi-uchi se encuentra limitado únicamente por la distancia, que ha de ser mínima para evitar que el adversario utilice sus armas principales de batalla, y por la sólida armadura que portaban los guerreros. A pesar de ello, existe un amplio abanico de técnicas eficaces, entre las que se incluyen especialmente ataques con cabeza, hombros y codos, tanto en su combate en pie como en suelo, derribos, luxaciones, estrangulaciones, etc.

\section{Tipos de kumi-uchi}

Sin embargo, aunque hasta ahora hemos hablado de que las características del kumi-uchi fueron desarrolladas inicialmente para enfrentarse a un guerrero provisto de armadura completa, en su evolución no se mantuvo dicho criterio. A lo largo del tiempo, muchas de las técnicas del kumi-uchi fueron extrapoladas al combate sin armadura, propio de otras realidades diferentes a la del campo de batalla (Draeger, 1996; Ratti \& W estbrook, 1994). Por tanto, diferenciaremos a partir de ahora el kumi-uchi como arte genérico, del yoroi kumi-uchi, específico con armadura. En algunas escuelas, por ejemplo la Yagyu Shingan Ryu, se denomina kachu yawara, correspondiendo kachu a "armadura" y yawara a "flexible, maleable".

Una vez que se prescinde de la armadura, el combate se amplía en muchos términos. Las zonas de impacto se multiplican y también las formas de impactar. De ahí que desarrollos posteriores añadiesen el estudio del atemi no gikko, 0 técnicas de impacto, que visaba el estudio de las diferentes formas características de impacto en cada arte en particular. Aquí, hemos de comprender que esta diferenciación cambió en muchos aspectos la evolución de las formas de combate. Con todo, como hemos señalado el kumi-uchi tiene sus propias formas de impacto, proyección, estrangulación, control, luxación, agarre, etc., y por tanto, es razonable que sus formas de desarrollo y aplicación sean completamente diferentes a las de otras formas de combate. Esto es lógico, pues cada arte tuvo sus propios orígenes y estaba condicionada por el entorno en que se vivía en cada periodo histórico y ante qué tipo de amenazas y defensas respondía.
EN EL KUMIUCHI, LAS ZONAS DE AGARRE ERAN MUY LIMITADAS POR EL YOROI, POR ELLO LAS FORMAS DE REALIZAR LOS ENCAJES Y CONTROLES DEBÍAN SER INGENIOSAS Y EXPLOTAR LAS LIMITACIONES INTRÍN SECAS. Así, LOS agARRES SE BASABAN EN SUJECIONES DE HOMBRO, CUELLO, BRAZO, CUERPO, ETC. (EN NINGÚN CASO A LAS SOLAPAS).

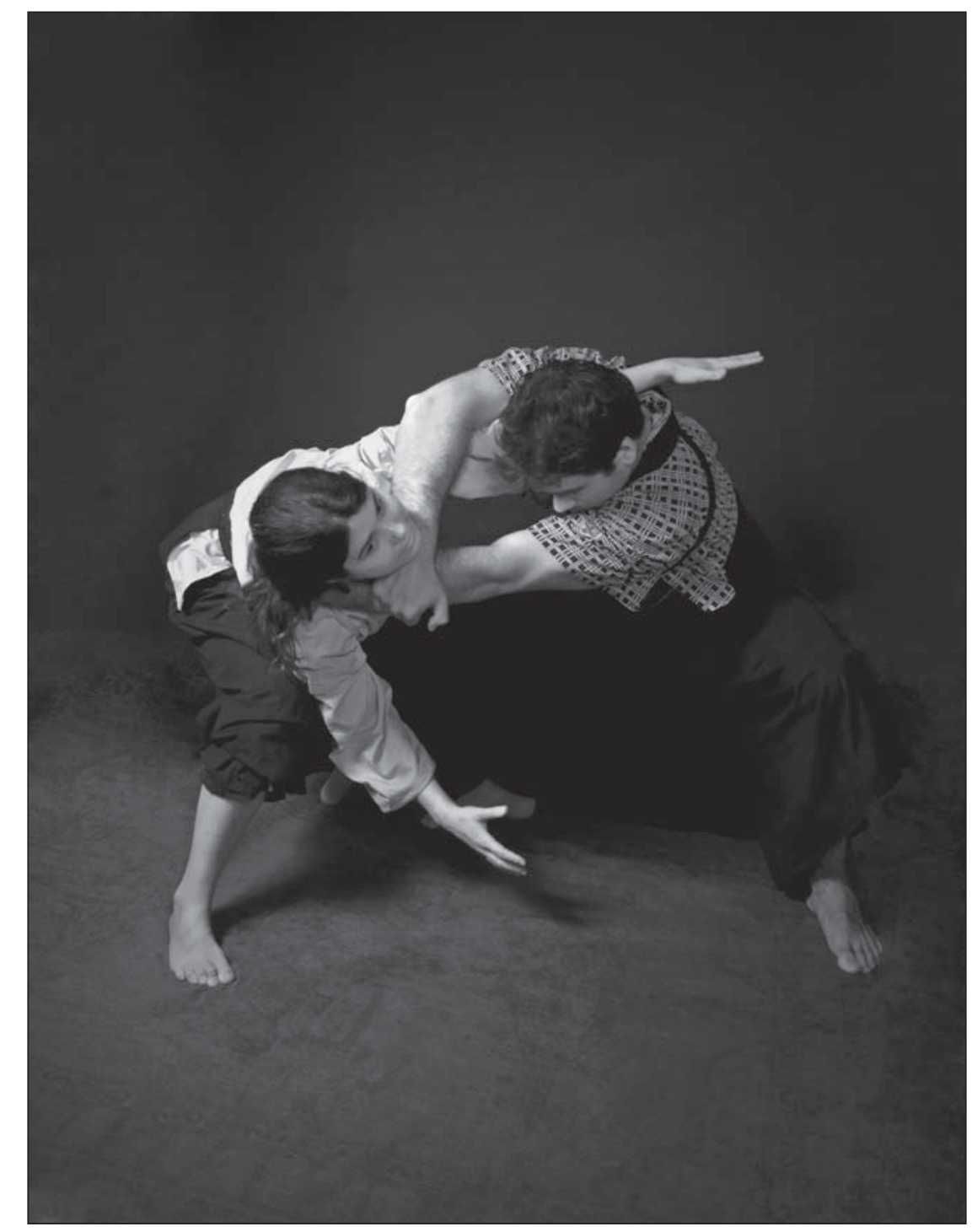


LA MECÁNICA DE LOS IMPACTOS EN KUMIUCHI VARÍA FRENTE A OTRAS ARTES CLÁSICAS, UTILIZANDO ELEMENTOS COMO LA CABEZA, EL HOMBRO O LAS CADERAS.

EN LA FOTOGRAFÍA EL ATACANTE SUJETA EL MENPO DEL ADVERSARIO MIENTRAS IMPACTA CON SU PUÑO SOBRE LA LÍNEA DE VISIÓN, EN UNA POSICIÓN CLÁSICA DE YOROI KUMIUCHI.
La Kaze no Ryu, como escuela tradicional, intenta mantener la esencia de las tradiciones heredadas, y por ello se esfuerza en transmitir la identidad particular de cada arte como sello y garantía de dicha manutención. En este sentido, el kumi-uchi antiguo de la Kaze no Ryu se divide en los tipos que muestra la Tabla 2 , en la que también se plasman algunos ejemplos de estudios de entrenamiento y formas propias de la escuela.

\begin{tabular}{|c|c|c|}
\hline Tipos & $\begin{array}{l}\text { Ejemplos de estudios de entrenamiento } \\
\text { (kihon, renshu...) }\end{array}$ & $\begin{array}{l}\text { Ejemplos de formas de } \\
\text { estudio (keitai/kata) }\end{array}$ \\
\hline $\begin{array}{l}\text { Koryu keitai } \\
\text { (forma } \\
\text { clásica) }\end{array}$ & $\begin{array}{l}\text { - Koryu ren (orientado al combate armado). } \\
\text { - Koryu to (orientado al combate contra espada). } \\
\text { - Koryu tsukamu (entrenamiento para la } \\
\text { aproximación y el agarre). } \\
\text { - Koryu shibaru (entrenamiento para apresar e } \\
\text { inmovilizar al adversario). }\end{array}$ & $\begin{array}{ll}\text { - } & \text { Iwanami } \\
\text { - } & \text { Iwagumo } \\
\text { - } & \text { Shoshu } \\
\text { - } & \text { Kaitei } \\
\text { - } & \text { Mujinto }\end{array}$ \\
\hline $\begin{array}{l}\text { Dento keitai } \\
\text { (forma } \\
\text { tradicional) }\end{array}$ & $\begin{array}{l}\text { - Dento ren (entrenamiento para el combate sin } \\
\text { armas) } \\
\text { - Dento Tsukamu (entrenamiento para el agarre } \\
\text { sin armas) } \\
\text { - Dento Shibaru (entrenamiento para el } \\
\text { aprisionamiento sin armas) }\end{array}$ & - Kaisui \\
\hline
\end{tabular}

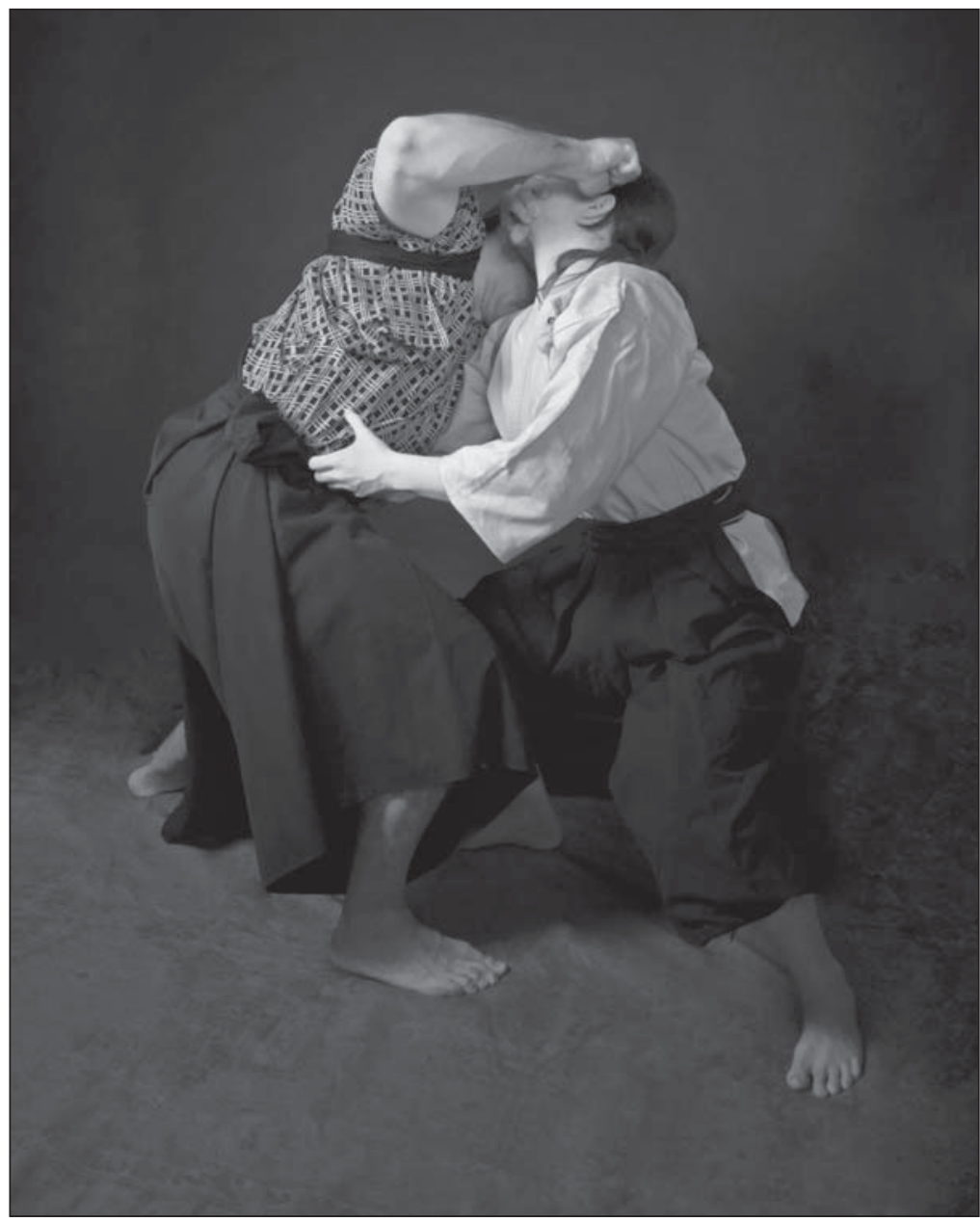

Antes de proceder al análisis de la Tabla 2, debemos reseñar que esta clasificación es una clasificación moderna del kumi-uchi. De hecho, la forma clásica de clasificarlo era en función de si se realizaba 0 no con yoroi, como se ha explicado al principio de este apartado. No obstante los maestros de los S. XIX y XX decidieron realizar una clasificación más moderna para lograr una mejor comprensión y canonización. Esta consistió en tres tipos: koryu keitai ${ }^{6}$, dento keitai ${ }^{7}$ (mostrados en la tabla) y shinten keitai. Como explicaremos, el shinten keitai es una forma moderna, y por ello no se ha incluido en la tabla anterior.

\footnotetext{
6 Habitualmente el término koryu suele traducirse como "escuelas clásicas". Realmente, ko significa "antiguo", pero el término ryu proviene del kanji "nagare" que se traduce como "fluir". Es por ello que la traducción más aproximada de koryu keitai sería "hacerlo fluyendo de la forma antigua".

7 Dento hace referencia a la tradición heredada.
} 
Keitai es un término único y característico de la escuela Kaze no Ryu Bugei. Su uso es un claro identificador de la herencia de la escuela como elemento de identidad. Keitai se compone de dos kanji:

\section{形 Kata, Kei, que se traduce como forma o figura. \\ 体 Tai, que se traduce como cuerpo, físico.}

Por tanto, keitai puede traducirse como "forma corporal", en referencia a que son formas de taijutsu.

Koryu keitai, que hemos traducido como "forma clásica", hace referencia a los ejercicios preparatorios y a las formas estudiadas en situación armada. Es decir, en las técnicas no sólo se utilizaba el propio cuerpo sino también armas diversas, generalmente de pequeño tamaño, aunque también era frecuente -como se aprecia en la tabla- el uso de la katana en dichas formas de combate.

Por el contrario, el dento keitai, que hemos traducido como "forma tradicional", hace referencia a los estudios y formas orientadas al combate de kumiuchi sin armas.

Finalmente, el shinten keitai, que podemos traducir como "forma evolucionada", son formas de aplicación desarrolladas en tiempos más modernos, sin la utilización de prendas ni elementos de combate clásicos. Por tanto, sería una adaptación de las formas de kumi-uchi a las situaciones históricas de los periodos posteriores a la restauración Meiji (a partir de 1866-1869

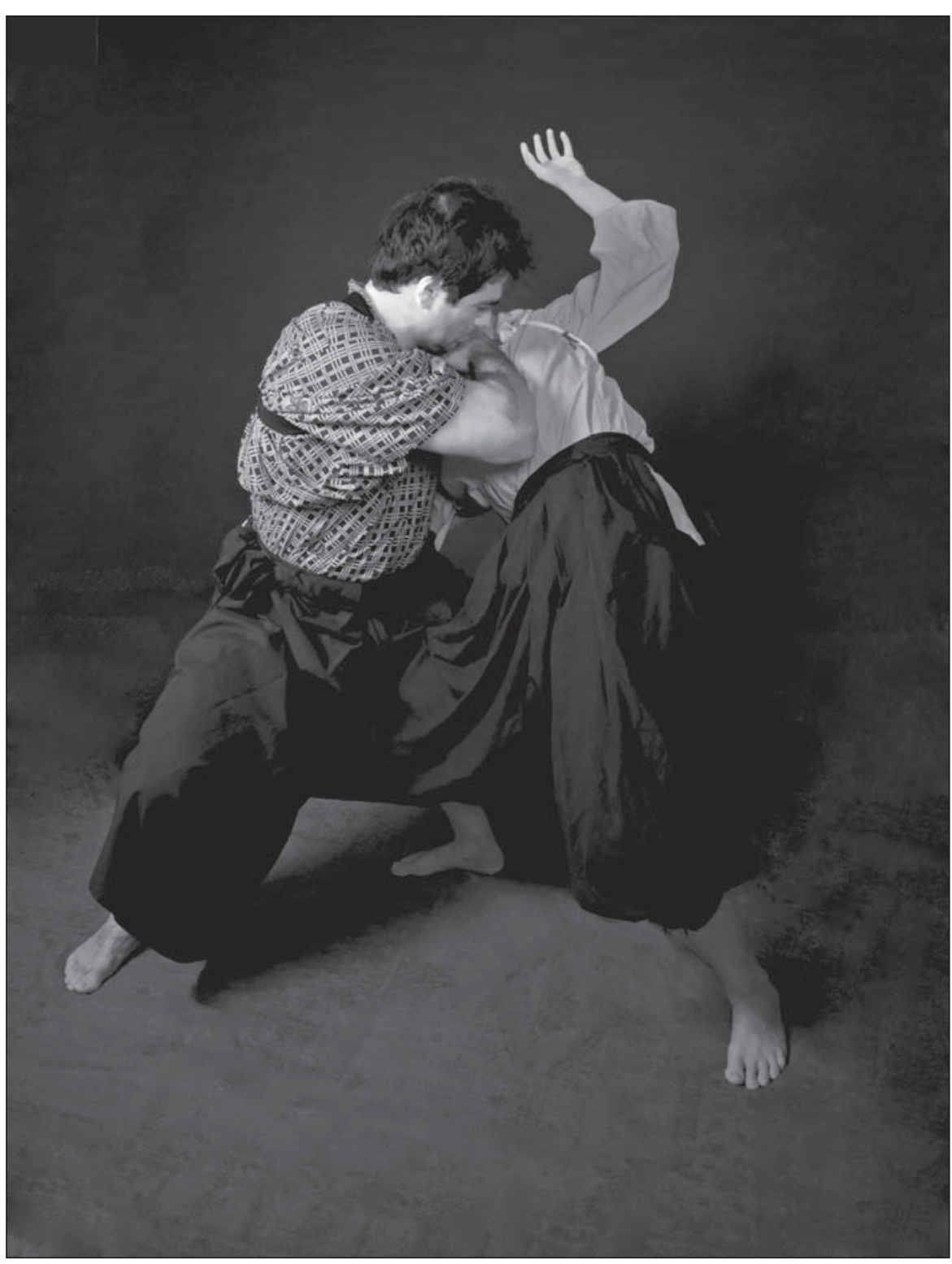

El uso de armas pequeñas, como en este caso un tanto sobre el cuello, en DOBLE APOYO, ERA AMPLIAMENTE DESARROLLADO EN LAS FORMAS DE KORYU KETAI. d.C.).

Entre los estudios de entrenamiento, si hablamos por ejemplo de koryu ren -siendo este ren "entrenamiento" o "práctica"-, hace referencia a las formas de combate libre de kumi-uchi con armas. Es decir, se parte del ataque y defensa 
libres, simulando una situación de combate realista. Aún así, dista mucho de los entrenamientos orientados al kakuto no bujutsu, o formas de guerra orientadas a la realidad, que entrenan la realidad del combate clásico sin reducirse al abanico técnico de una sola disciplina.

Cada estudio de entrenamiento se fundamenta en una serie de elementos principales. Por ejemplo, en el caso del koryu shibaru son los siguientes:

- Keten mi shibaru, o estudio de los puntos vulnerables;

- Koryu uchi, forma clásica de estudio de las formas de impactar en el oponente;

- Koryu keten mi, orientado a alcanzar al oponente en lugares estratégicos para obtener ventaja y dañar al oponente ${ }^{8 ;}$

- Koryu kamae, referido al estudio de las posturas clásicas de combate; y

- Koryu henka, como formas de encadenamiento de técnicas.

Por otro lado, si nos centramos en el estudio de keitai, recordando que nos referimos a las formas características de Kaze no Ryu de formas preestablecidas y heredadas, vemos que cada keitai aporta las identidades propias de cada periodo y condición particular. De hecho, cada keitai es en sí mismo un núcleo de estudio propio, pues es la herencia que lega la escuela y en ella está inscrita su historia y sus conceptos prácticos de combate. Las visiones son tremendamente variadas e inteligentes. Parten de situaciones totalmente dispares, ya sea haciendo uso específico de confrontaciones cuchillo contra cuchillo, o haciendo uso de la katana como elemento de agarre, apoyo e impacto.

Un caso muy particular es el keitai llamado kaisuig que debe su peculiar nombre ("agua de mar") a su origen. Ideado en combates en los que el agua, con una profundidad suficiente, permitía realizar técnicas orientadas al ahogamiento final. Este keitai desarrolla formas únicas de control final usando controles dominando el "hara" con la combinación del uso de brazo y pierna para conseguir apresar a uke (el contrario), estrangulaciones combinadas con luxaciones cervicales, así como una variedad enorme de luxaciones sobre la espalda y caderas.

El uso de proyecciones de mano sobre las piernas es otra de sus características. No debemos olvidar, que para el desarrollo de este keitai ambos oponentes realizan las técnicas sin armadura, lo que permite el uso de agarres e impactos con cabeza, hombros, rodillas y talones. Además, este keitai introduce en algunos aspectos las formas de combate de katame no gikoo (combate en suelo) específicas del kumi-uchi, que suponen uno de los tesoros heredados de este arte en particular.

8 Algunos ejemplos de estos lugares estratégicos son shogo me (meridiano de los ojos), shogo kokyu (meridiano de la respiración), shogo heiko (meridiano del equilibrio) o shogo shincho (meridiano de la estabilidad mental).

9 Kai significa "mar" u "océano" y sui/mizu significa "agua". Por tanto significa "agua del mar". 


\section{Dimensión del kumi-uchi}

Más allá de profundizar en aspectos y detalles técnicos, que nos llevarían a una dimensión de estudio mucho más extensa que la que se pretende en este trabajo, debemos subrayar que el desarrollo de las técnicas de kumiuchi estuvo claramente apoyado sobre situaciones clásicas de conflicto, siendo estas las motivaciones per se de su creación y uso. Desde esta perspectiva tradicional y purista, resulta ilógico e incluso aberrante pensar en un kumi-uchi que se distancie de las dos razones de su existencia: la concepción clásica del combate y la eficacia de su aplicación en las situaciones para las que fue desarrollado.

En las artes guerreras 0 bugei, y especialmente en el kumi-uchi, la idea de transitar en el delgado filo de una katana debe estar presente en toda práctica. A un lado se sitúa el espacio de la vida, al otro lado el de la muerte. El momento presente define el peso de la balanza, y solo tendremos este preciso instante para poder definir la situación. El después siempre es tarde. No hablamos de otra cosa que del ichi go ichi e, "un instante, una acción"10.

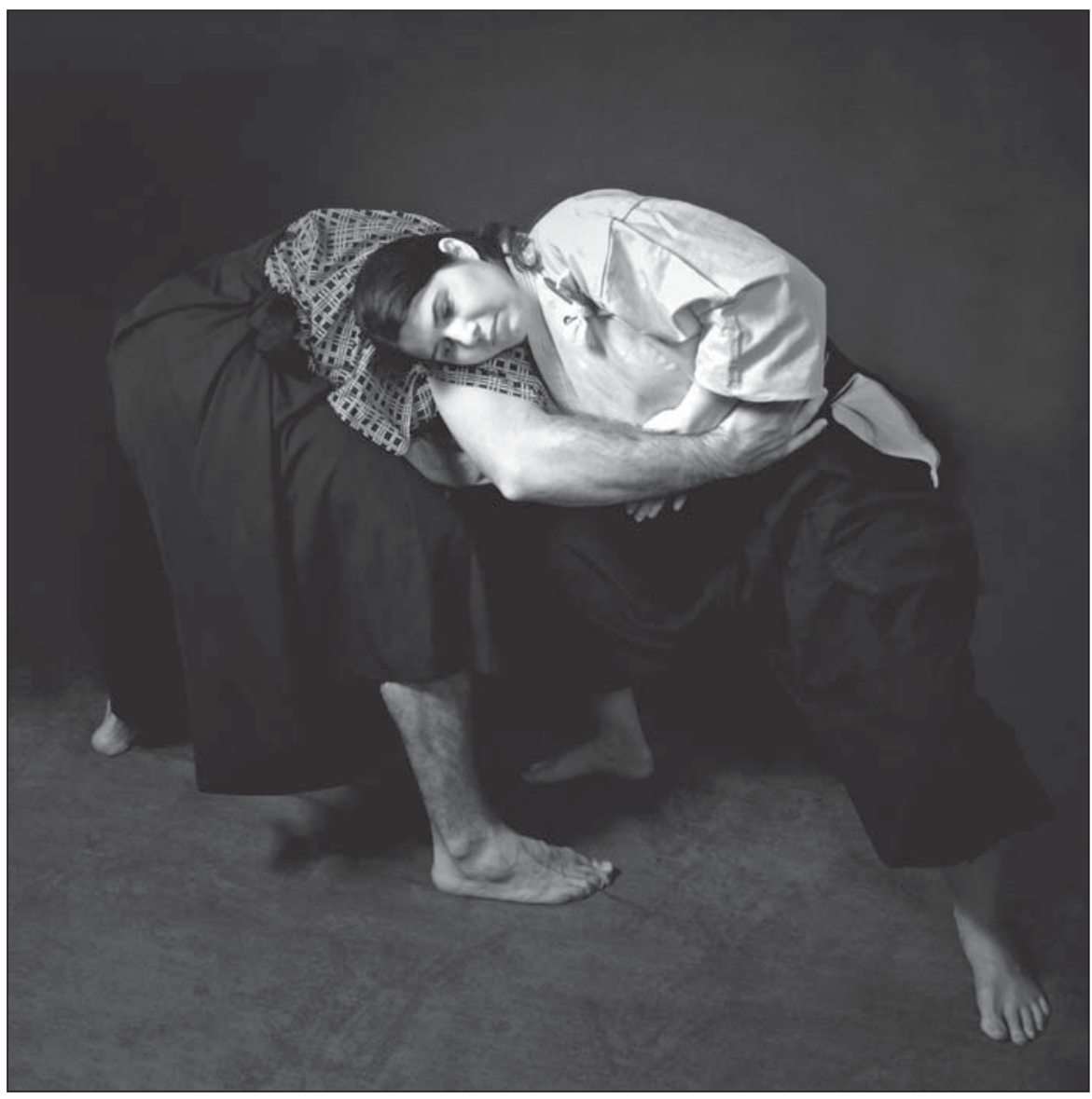

Los movimientos de paso de mano buscan el SUbterfugIO de Un FérReO ABRAZO para buscar estratéGICAMENTE agarRes y posicion ES VENTAJOSAS. EN ESTA TÉCNICA SE ILUSTRA UNO DE LOS MOVIMIENTOS CARACTERÍSTICOS DE LA SETEGATA DENOMINADA IWANAMI.

La importancia clave del ichi go ichi e es enseñada como parte fundamental desde el primer momento de estudio. Las seiteigata ("formas en orden correcto" ${ }^{11}$ ) denominadas iwanami/iwagumo (ola sobre la roca/nube sobre la roca) como introducción básica al kumi-uchi muestran claramente este principio, especialmente

\footnotetext{
10 Se trata de una locución japonesa muy usada para definir la importancia del momento, y es uno de los conceptos filosóficos básicos y fundamentales que se transmiten en el bugei.

11 Seiteigata se compone de tres kanjis. Sei significa "correcto". Tei significa "orden", y kata/ gata significa "forma". Es decir, "formas en orden correcto". Se trata de la forma de denominar a la clasificación que se realizó de las katas para su estudio.
} 
en las formas de iwagumo, donde el ma-ai (distancia), la postura (kamae) y todos y cada uno de los detalles específicos que se enseñan en la kata definen ese instante único que permitirá vivir o morir.

La dicotomía entre la vida y la muerte fue un principio muy presente en la tradición japonesa. Existen evidencias históricas que narran la especial dedicación que tenía la clase samurái a cultivar el desapego a la vida (véase por ejemplo Ratti \& W estbrook, 1994). Este desapego está claramente caracterizado y desarrollado en cada movimiento del kumi-uchi. Sin embargo, no podemos quedarnos con esta idea como una simple indiferencia hacia la muerte, que es posible el concepto occidental más extendido, sino que se trata de una doctrina claramente vitalista, pero fuertemente marcada por los ideales de servicio ${ }^{12}$ y honor. Desde esta perspectiva no podemos catalogar al kumi-uchi como un arte brutal y carente de interés moderno. El estudio de sus particularidades e identidad, desde la concepción histórica del combate y todo lo que lo envuelve, así como el origen de la cultura castrense japonesa personificada en el bun bu ryo $\mathrm{do}^{13}$, aporta una riqueza única y valiosa como herencia a valorar y mantener.

\section{Evolución del kumi-uchi}

Como producto cultural vivo, y en función de las transformaciones sociales y culturales del pueblo japonés, el kumi-uchi fue adaptándose a cada época, manteniendo sus características como arte de combate que salvaguardaba la vida de forma complementaria al resto de disciplinas militares. En su aplicación a ámbitos no miliares, esto es, al margen de la guerra, hizo replantear muchas de las formas del kumi-uchi. Ahora los ataques ya no se producían por guerreros armados y equipados en un campo de batalla, sino entre elementos sociales heterogéneos y en situaciones diversas de conflicto. Esas nuevas formas empleadas para resolver los conflictos de carácter cotidiano recibieron el nombre de jujutsu.

Anteriormente hemos aludido a la denominación yawara, que corresponde a su vez y precisamente al kanji "ju". Ju puede ser traducido por "flexible, maleable, blando", y jutsu por "arte, técnica o habilidad". Literalmente, jujutsu puede traducirse como "arte blando" o "arte flexible" (Ratti \& W estbrook, 1994). Esta denominación, lejos de su apariencia extraña para denominar un eficaz sistema de combate, dice mucho de sus principios fundamentales y prácticas, pero a su vez puede llevar a malentendidos. Sin pretender acabar el tema, y siendo conscientes de su amplia dimensión, señalaremos que mientras el kumi-uchi aportaba la idea de encuentro de fuerzas en oposición, el jujutsu incorporaba la idea de cesión y acompañamiento de la fuerza. Las fuerzas ya no buscaban el impacto directo, la

12 Ha de recordarse que el término samurái proviene del verbo japonés que significa "servir".

13 Bun bu ryo do es un concepto muy utilizado en bugei para ilustrar al estudiante la importancia del equilibrio entre cuerpo y mente. Bun significa "cultura", bu "guerra", ryo es traducido por "ambos" y finalmente do proviene de la raíz michi, esto es, "camino", pero que al cambiar a la pronunciación do adquiere un sentido más espiritual, generalmente traducido por camino espiritual o vía de desarrollo personal. Por tanto, su traducción más correcta sería: "En la cultura de la guerra existen ambos caminos". Estos caminos son las formas de desarrollo físico y de combate, y las formas de desarrollo mental y espiritual. 
confrontación, sino que estas, sin dejar de ser fuerzas, se armonizaban para lograr daños mayores.

Es por ello que el jujutsu bebe en gran medida del kumi-uchi. Por un lado, del abanico técnico proporcionado por el kumi-uchi en cuanto a impactos, proyecciones, controles, etc. En muchas de las katas más antiguas de jujutsu puede sentirse la esencia del kumi-uchi que hemos tratado de transmitir en este artículo. Por otra parte, el kumi-uchi proveyó de muchos de los fundamentos que permitieron el desarrollo de esa nueva concepción del combate cuerpo a cuerpo. Si el jujutsu pudiera ser comparado con las etapas de la vida del hombre, se correspondería con la edad adulta, mientras que el kumi-uchi sería la etapa de infancia y crecimiento. Posteriormente, el jujutsu también evolucionó, y en su madurez tomó entre otras las formas, como el aikijujutsu ("arte blando con el uso de la unión de la energía) y el koppojutsu ("arte del método de los huesos", empleado especialmente para lograr la fractura de los mismos), que también forman parte del estudio de la Kaze no Ryu.

\section{Conclusión}

Este breve estudio ha pretendido exponer las bases fundamentales del kumiuchi, sus tipos, evolución y otras características desde la perspectiva de la Kaze no Ryu. A pesar de parecer un arte primitivo y superado por artes más modernas, no podemos caer en la tentación de minusvalorarlo. El kumi-uchi, como todas las artes que se incluyen en el bugei, encierra una cantidad de detalles que pueden pasar desapercibidos al lego, pero que son la base para el entendimiento de principios y técnicas marciales que trascienden el propio kumi-uchi. El estudio del kumi-uchi puede extenderse durante muchos años, y a pesar de tener una cantidad de katas muy inferior a sus artes hermanas, su amplitud y capacidad de desarrollo debe tener una consideración especial dentro de la construcción de una mentalidad adecuada para el entendimiento de las técnicas de combate estudiadas del taijutsu.

\section{AGRADECIMIENTOS}

Un especial agradecimiento a Shidoshi Jordan Augusto y a Sensei Juliana Galende por sus enseñanzas en la tradición de la Kaze no Ryu. Gran parte de los contenidos de este artículo han podido elaborarse gracias a sus enseñanzas, así como a los materiales de estudio y fondos de archivo de la Sociedad Brasileira de Bugei (www.bugei.com.br) y la European Bugei Society (www.bugei.eu).

\section{REFEREN CIAS}

Augusto, J. \& Galende, J. (s.f.). Bujutsu. A arte e o Espirito da Guerra. Goiânia (Brasil): Kanji.

Draeger, D.F. (1996). The Martial Arts and Ways of Japan. Volumen I: Classical Bujutsu. N ew York: Weatherhill.

Friday, K.F. \& Humitake, S. (1997). Legacies of the Sw ord: The Kashima-Shinryu and Samurai Martial Culture. Honolulu: University of Hawaii Press. 
Gatling, L. (2010). Jujutsu. En T.A. Green \& J.R. Svinth(Eds.). Martial arts of the world: An Encyclopedia of History and Innovation. Vol. I. (pp. 120-126). Santa Barbara, CA: ABC-CLIO.

Mol, S. (2001). Classical Fighting Arts of Japan: A Complete Guide to Koryu Jujutsu. Tokyo/N ew York/London: Kodansha.

Mol, S. (2003). Classical Weaponry of Japan: Special Weapons and Tactics of the Martial Arts. Tokyo: Kodansha.

Ratti, 0.\& Westbrook, A. (1994). Los Secretos del Samurai. Las artes marciales en el Japón Feudal. Madrid: Alianza Deporte.

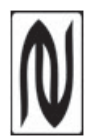

